\title{
Prediction of Re-positivity for Coronavirus Nucleic Acid Among COVID-19 Patients in the Recovery Phase
}

\section{OPEN ACCESS}

Edited by:

Longxiang Su,

Peking Union Medical College Hospital (CAMS), China

Reviewed by: Dejan Baskic,

University of Kragujevac, Serbia

Yuhan Xing,

The Chinese University of Hong

Kong, China

*Correspondence:

Shao-wei L

li_shaowei81@hotmail.com

tThese authors have contributed equally to this work and share first authorship

Specialty section: This article was submitted to Infectious Diseases - Surveillance, Prevention and Treatment. a section of the journal Frontiers in Medicine

Received: 23 October 2020 Accepted: 26 February 2021 Published: 05 May 2021

Citation:

Zhu S-f, Sun B, Li J-k, Cai Y, Li P-f,

Hong J-c, Li J-h, Xu S-w, Li X-y, Xue C-w, Gu B-b, Wu J-f, Zhou X-b, Suo H, Duan P-I, Wu X-X and Li S-W (2021) Prediction of Re-positivity for

Coronavirus Nucleic Acid Among COVID-19 Patients in the Recovery

Phase. Front. Med. 8:620727. doi: 10.3389/fmed.2021.620727

\begin{abstract}
Shu-fen Zhu ${ }^{1 \dagger}$, Bo Sun ${ }^{2 \dagger}$, Jin-kuang $\mathrm{Li}^{3}$, Yue Cai ${ }^{4}$, Peng-fei Li ${ }^{5}$, Ji-chang Hong ${ }^{6}$, Jin-hai $\mathrm{Li}^{7}$, Shi-wen $\mathrm{Xu}^{8}{ }^{8}$, Xiao-yang $\mathrm{Li}^{9}$, Chen-wei Xue ${ }^{9}$, Bin-bin $\mathrm{Gu}^{4}$, Jian-fen $\mathrm{Wu}^{4}$, Xian-bin Zhou ${ }^{4}$, Hong Suo ${ }^{10}$, Pei-lin Duan ${ }^{1}$, Xin-xin Wu ${ }^{11}$ and Shao-wei $\mathrm{Li}^{4,12 \star}$

${ }^{1}$ Department of Critical Care Medicine, The Affiliated Hospital of Inner Mongolia Medical University, Hohhot, China, ${ }^{2}$ Department of Brain Surgery, Zhongxiang People's Hospital, Jinmen, China, ${ }^{3}$ Department of Radiology, Zhongxiang People's Hospital, Jinmen, China, ${ }^{4}$ Department of Gastroenterology, Taizhou Hospital of Zhejiang Province Affiliated to Wenzhou Medical University, Taizhou, China, ${ }^{5}$ Inner Mongolia People's Hospital, Hohhot, China, ${ }^{6}$ Department of Critical Care Medicine, Zhongxiang People's Hospital, Jinmen, China, 'Department of Neurology, Zhongxiang People's Hospital, Jinmen, China, ${ }^{8}$ Taizhou Hospital of Zhejiang Province Affiliated to Wenzhou Medical University, Taizhou, China, ${ }^{9}$ Zhongxiang People's Hospital, Jinmen, China, ${ }^{10}$ Department of Respiratory Medicine, The Affiliated Hospital of Inner Mongolia Medical University, Hohhot, China, ${ }^{11}$ Inner Mongolia Medical University, Hohhot, China, ${ }^{12}$ Key Laboratory of Minimally Invasive Techniques \& Rapid Rehabilitation of Digestive System Tumor of Zhejiang Province, Taizhou Hospital of Zhejiang Province Affiliated to Wenzhou Medical University, Linhai, China
\end{abstract}

Background and Objectives: Although the pathogenesis and treatment of coronavirus disease 2019 (COVID-19) have been gradually revealed, the risk for re-emergence of coronavirus nucleic acids in recovered patients remains poorly understood. Hence, this study evaluated the risk predictors associated with re-positivity for virus nucleic acid.

Methods: Between February 1 and March 20, 2020, we retrospectively reviewed the clinical epidemiological data of 129 COVID-19 patients who were treated at Zhongxiang People's Hospital of Hubei Province in China. Subsequently, a risk prediction model for the re-positivity of virus nucleic acid was developed, and a receiver operating characteristic (ROC) curve was drawn for further validation.

Results: In this study, the rate of re-positivity for virus nucleic acid was $17.8 \%$ (23/129) where all re-positivity cases were asymptomatic. The median time interval from nucleic acid re-positivity to discharge after being cured again was 11.5 days (range: 7-23 days). Multivariate logistic regression analysis showed that leukocytopenia [odds ratio (OR) 7.316, 95\% confidence interval (Cl) 2.319-23.080, $p=0.001$ ], prealbumin < $150 \mathrm{mg} / \mathrm{L}$ (OR 4.199, 95\% Cl 1.461-12.071, $p=0.008$ ), and hyperpyrexia (body temperature $>39^{\circ} \mathrm{C}$, OR $4.643,95 \% \mathrm{Cl} 1.426-15.117, p=0.011$ ) were independent risk factors associated with re-positivity. The area under the ROC curve was 0.815 (95\% Cl, 0.729-0.902).

Conclusion: COVID-19 patients with leukocytopenia, low prealbumin level, and hyperpyrexia are more likely to test positive for virus nucleic acid after discharge. Timely and effective treatment and appropriate extension of hospital stays and quarantine periods may be feasible strategies for managing such patients.

Keywords: COVID-19, re-positivity, virus nucleic acid, risk predication, re-emergence of coronavirus nucleic acids 


\section{INTRODUCTION}

In early December 2019, the first case of unexplained coronavirus pneumonia was reported in Wuhan, China (1), which was followed by an outbreak worldwide. In January 2020, Ren et al. (2) led the completion of whole-genome sequencing of the coronavirus and confirmed a homology of more than $85 \%$ with bat severe acute respiratory syndrome (SARS)-like coronavirus (bat-SL-CoVZC45). Therefore, the International Virus Classification Committee (ITCV) named it as SARS coronavirus 2 (SARS-CoV-2), which was then officially named as coronavirus disease 2019 (COVID-19) (3). With increasing research being conducted concerning pathogen transmission and mechanisms $(4,5)$, continuous update of guidelines on treatment and diagnosis $(6,7)$, and its prevalence has now been controlled and effectively mitigated in China.

Serious dangers concerning the frequent emergence of test repositivity of virus nucleic acid in recovered COVID-19 patients have been a widespread concern (8-10). Some studies revealed that this rate ranges from 3.3 to $30.8 \%(9-13)$. Yuan et al. (13) reported that young patients ( $<18$ years old) had much higher re-positivity rates $(30.8 \%)$ than those aged $\geq 18$ years (9.5\%). However, its mechanism remains unclear and necessitates further research. Of note, most re-positive patients do not show infectivity, which excludes the possibility of simple viral relapse or secondary infection (13-15). A few recent studies have proposed that virology, the detection of specimens, or the patient' s condition might be potential reasons for test re-positivity of virus nucleic acid (16-18).

In this study, we retrospectively analyzed clinical epidemiological data of 129 COVID-19 patients, and evaluated the risk factors associated with re-positivity for virus nucleic acid. Similarly, prompt and effective treatment and appropriate extension of hospitalization and quarantine period may be feasible strategies for patient management.

\section{MATERIALS AND METHODS}

\section{Patients}

This retrospective study, which complied with the Declaration of Helsinki, was approved by the Ethics Committee of Zhongxiang People's Hospital (ZXRY20200420) and the Ethics Committee of The Affiliated Hospital of Inner Mongolia Medical University (WZ 2020033).

Based on the novel coronavirus pneumonia diagnosis and treatment scheme (Trial Version 4), COVID-19 patients do not only require a clear epidemiological history and clinical manifestations but also must meet at least one of the following conditions: (1) positivity for coronavirus nucleic acid on realtime fluorescent reverse transcription-polymerase chain reaction (RT-PCR); (2) a viral gene sequence showing high homology with the novel coronavirus; and (3) positivity for serum novel coronavirus-specific IgM and IgG antibodies. Furthermore, exclusion criteria were as follows: death due to COVID-19 $(n=$ $1)$ or other diseases [acute cardiovascular disease $(n=2)$, acute renal failure $(n=1)$ ], loss to follow-up after being transferred to another hospital $(n=1)$, and serious loss of clinical data $(n$
=1). Finally, 129 COVID-19 cases were included in our study (Figure 1).

\section{Study Design}

Between February 1 and March 20, 2020, we retrospectively analyzed the demographic, clinical, and epidemiological data of 129 COVID-19 patients admitted to our institution. Laboratory findings, radiological results, and therapy course were independently obtained from a prospectively maintained database in Zhongxiang People's Hospital.

Patients were divided into two groups: those with re-positivity $(n=23)$ and without re-positivity $(n=106)$ for the virus nucleic acid group. The differences in sex, age, comorbidities, white blood cell count, body temperature, and prealbumin levels between the two groups were compared, and a risk prediction model was established via multivariate analysis. A receiver operating characteristic (ROC) curve was drawn to validate the model.

Chest computed tomography (CT) results were reviewed by two physicians (Shu-fen Zhu, Pei-lin Duan) and a radiologist (Jin-kuang Li). Leukopenia was defined as a white blood cell count $<4 \times 10^{9} / \mathrm{L}$. Hyperpyrexia was defined as body temperature above $39^{\circ} \mathrm{C}$. The follow-up outcomes were collected through electronic medical records or telephone interviews by referral physicians or patients with a deadline of until March 20, 2020.

\section{SARS-CoV-2 Nucleic Acid Testing}

RT-PCR was used to detect novel coronavirus nucleic acids. The detection equipment were GeneRotex96 automatic nucleic acid extraction and Gentier96E real-time fluorescent quantitative PCR instruments (Tianlong Technology Co., Ltd., Xi'an, China). The extraction reagent used was the virus DNA/RNA extraction kit (magnetic beads method) (Tianlong Technology Co., Ltd., $\mathrm{Xi}$ 'an, China). The detection reagent was the SARS-CoV-2 nucleic acid detection kit (PCR probe method) (Da'AN Gene Co.,

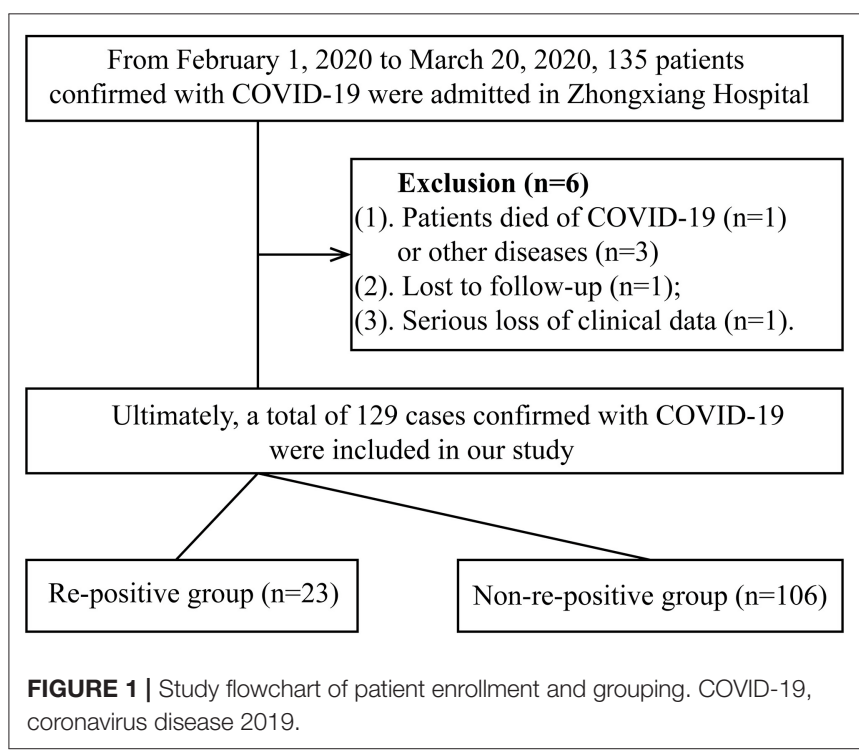


Ltd., Zhongshan, China and Shengxiang Biotechnology Co., Ltd., Hunan, China). The target genes were the ORF1ab and $\mathrm{N}$ genes in the SARS-CoV-2 genome, and the positive reading criteria were described in the reagent kit packaging. Specimen sampling, nucleic acid preparation, and amplification were performed strictly using the kit instructions. In case of suspicious results, re-sampling and review were required.

\section{Clinical Cure Standards for COVID-19 and Re-positivity of Nucleic Acid and Serum Antibody}

The standard clinical cure for COVID-19 patients was in reference to the Novel Coronavirus Pneumonia Diagnosis and Treatment Scheme (Trial Version 4): (1) normal body temperature for more than 3 days; (2) significant improvement of respiratory symptoms; (3) notable absorption of inflammation on pulmonary imaging; and (4) two consecutive negative for SARS-CoV-2 nucleic acid tests (sampling interval of at least $24 \mathrm{~h}$ ). When the patients met these criteria, they were quarantined in a designated area to continue isolation in observation and rehabilitation treatment for at least 14 days. During this period, upper respiratory specimens and blood specimens were collected for a SARS-CoV-2 nucleic acid and serum antibody test on day 14.

\section{Statistical Analyses}

Continuous variables were summarized as mean \pm standard deviation (SD) or median plus interquartile range (IQR), and categorical variables were expressed as frequencies and percentages. In the univariate analysis, continuous and categorical variables were assessed via Student's $t$-test (for those

TABLE 1 | Clinical epidemiological characteristics and a comparative analysis between the re-positivity and non-re-positivity group.

\begin{tabular}{|c|c|c|c|c|}
\hline & Total $(n=129)$ & Re-positivity $(n=23)$ & Non-re-positivity (106) & $P$-value \\
\hline Age (years), mean \pm SD & $51.6 \pm 14.0$ & $48.9 \pm 10.1$ & $52.2 \pm 14.7$ & 0.299 \\
\hline Sex, male, $n(\%)$ & $69(53.5)$ & $11(47.8)$ & $58(54.9)$ & 0.176 \\
\hline Diabetes & $9(7.0)$ & $0(0.0)$ & $9(8.5)$ & 0.361 \\
\hline Hypertension & $16(12.4)$ & $4(17.4)$ & $12(11.3)$ & 0.485 \\
\hline CKD & $7(5.4)$ & $1(4.3)$ & $6(5.7)$ & 0.801 \\
\hline Malignant diseases & $5(3.9)$ & $1(4.3)$ & $4(3.8)$ & 0.897 \\
\hline Antibiotics, $n(\%)$ & $92(71.3)$ & 19 (82.8) & 73 (68.9) & 0.187 \\
\hline Glucocorticoid, $n$ (\%) & $40(31.0)$ & $6(26.1)$ & 34 (32.1) & 0.629 \\
\hline Bilateral pneumonia, $n$ (\%) & $83(64.3)$ & $19(82.6)$ & $64(60.4)$ & 0.044 \\
\hline$>39.0^{\circ} \mathrm{C}$ & $24(18.6)$ & $9(39.1)$ & $15(14.2)$ & 0.014 \\
\hline Leukocyte $\left(\times 10^{9} / \mathrm{L}\right)$, mean \pm SD & $4.9 \pm 2.0$ & $3.4 \pm 0.9$ & $5.3 \pm 2.1$ & $<0.001$ \\
\hline Leukopenia, $n(\%)$ & $54(41.9)$ & $18(78.3)$ & $36(34.0)$ & $<0.001$ \\
\hline Neutrophil ratio (\%), mean \pm SD & $65.8 \pm 12.3$ & $68.0 \pm 7.8$ & $65.3 \pm 13.1$ & 0.207 \\
\hline Lymphocyte ratio (\%), mean \pm SD & $23.7 \pm 11.0$ & $21.0 \pm 8.1$ & $24.2 \pm 11.5$ & 0.121 \\
\hline Monocyte ratio (\%), mean \pm SD & $6.3 \pm 3.5$ & $7.2 \pm 3.2$ & $6.1 \pm 3.5$ & 0.163 \\
\hline CRP (mg/L), mean \pm SD & $33.5 \pm 24.5$ & $29.0 \pm 13.7$ & $34.9 \pm 26.8$ & 0.168 \\
\hline $\mathrm{ESR}(\mathrm{mm} / \mathrm{H})$, mean $\pm \mathrm{SD}$ & $28.8 \pm 29.4$ & $28.9 \pm 37.5$ & $28.8 \pm 27.5$ & 0.981 \\
\hline $\mathrm{PCT}(\mathrm{ng} / \mathrm{ml})$, mean $\pm \mathrm{SD}$ & $0.5 \pm 0.6$ & $0.3 \pm 0.2$ & $0.5 \pm 0.7$ & 0.112 \\
\hline $\mathrm{LDH}(\mathrm{U} / \mathrm{L})$, mean $\pm \mathrm{SD}$ & $215.7 \pm 86.3$ & $207.2 \pm 48.6$ & $217.3 \pm 92.0$ & 0.632 \\
\hline Albumin (g/L), mean $\pm S D$ & $41.4 \pm 21.6$ & $39.7 \pm 5.8$ & $41.7 \pm 23.7$ & 0.685 \\
\hline
\end{tabular}

$S D$, standard deviation; $C C D$, chronic cardiovascular disease; $C R D$, chronic respiratory disease; $C K D$, chronic kidney disease; ESR, erythrocyte sedimentation rate.

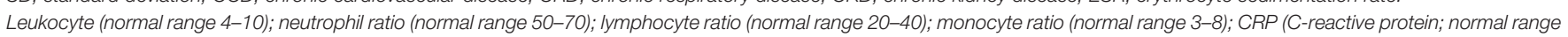

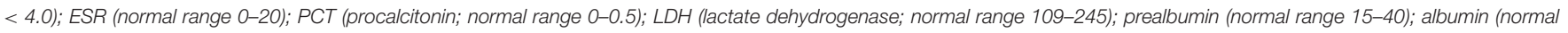
range 35-55); ALT and AST (alanine aminotransferase and Alanine aminotransferase; normal range 0-40); CK (creatinine kinase; normal range 18-198). 
with a normal distribution) or Wilcoxon signed-rank test (for those with an abnormal distribution) and chi-squared test, respectively. A $p<0.05$ was considered statistically significant.

Binary logistic regression was performed to develop the risk prediction model for re-positivity of SARS-CoV-2 nucleic acid, and the ROC curve was used to validate the model. The Statistical Package for the Social Sciences (SPSS, version 23.0; IBM Corp., Armonk, NY, USA) was used for data analysis.

\section{RESULTS}

\section{Epidemiological History of COVID-19 Patients}

A total of 129 COVID-19 patients were retrospectively analyzed. The following findings were gathered: 65 cases (51.9\%) with travel or living histories in or around the Wuhan epidemic area; 42 cases $(32.6 \%)$ with clear contact histories with COVID-19 patients; 10 cases $(7.8 \%)$ living with COVID-19 patients in the same building, but denying a history of contact; five cases $(3.9 \%)$ working in the same company as a COVID-19 patient; and five cases $(3.9 \%)$ with no clear history of contact. Hypertension was found in 16 cases, diabetes in nine cases, chronic cardiovascular disease in nine cases, chronic kidney disease in seven cases, chronic respiratory diseases in six cases [bronchial asthma $(n=$ $1)$, bronchiectasis $(n=1)$, chronic bronchitis $(n=1)$, chronic obstructive pulmonary disease $(n=3)$ ], and malignant diseases in five cases (Table 1). The time interval from discharge to repositivity for coronavirus nucleic acid and serum antibody was 14 days.

\section{Demographic and Clinical Characteristics of COVID-19 Patients}

All 129 patients were diagnosed with COVID-19 based on a positive nasopharyngeal swab nucleic acid test. The rate of re-positivity for virus nucleic acid was $17.8 \%(23 / 129)$ with a mean age of $51.5 \pm 14.2$ years. The proportion of male $(54.3 \%)$ and female $(45.7 \%)$ cases was approximately equal. Pneumonia, suggested by radiography, was detected in 109 cases $(84.5 \%)$, where $80(62.0 \%)$ of them presented with bilateral pneumonia. There were 87 cases $(67.4 \%)$ with fever on admission, mainly of low or medium severity. During hospitalization, antiviral drugs (ribavirin, peginterferon, and/or abidor) and immunomodulatory drugs (thymosins) were administered for all COVID-19 patients. Similarly, there were $71(70.5 \%)$ and 40 patients $(31.0 \%)$ who received antibiotics and glucocorticoids during hospitalization, respectively.

\section{Predictive Factors Associated With Re-positivity for Virus Nucleic Acid: Findings of Univariate and Multivariate Analyses}

The results of the univariate analysis for possible predictive factors associated with the re-emergence of virus nucleic acids are summarized in Table 2. The following four variables were determined to be significant risk factors according to a univariate analysis $(p<0.05)$ : leukopenia, prealbumin $<150 \mathrm{mg} / \mathrm{L}$, hyperpyrexia, and bilateral pneumonia. In the binary logistic regression analysis, leukopenia [odds ratio (OR) 7.316, 95\% confidence interval (CI) 2.319-23.080, $p<0.001$ ], prealbumin $<150 \mathrm{mg} / \mathrm{L}$ (OR 4.199, 95\% CI 1.461-12.071, $p=0.035$ ), and hyperpyrexia (OR 4.643, 95\% CI 1.426-15.117, $p=0.035$ ) were independent risk predictors associated with re-positivity for SARS-CoV-2 RNA.

On this prediction model, an ROC curve was drawn to validate the model. The area under the ROC curve was 0.815 (95\% CI 0.729-0.902), which suggested that the model had moderate to good predictive accuracy (Figure 2).

\section{Clinical Outcomes After Re-positivity for Coronavirus Nucleic Acid}

All re-positive patients were asymptomatic, and the emergence of new pulmonary infiltration or consolidation was not revealed on chest CT. Among the 23 re-positive cases, five were discovered during a community medical examination after isolation had been lifted. The family members of these five patients were also quarantined, and all coronavirus nucleic acid tests were found to be negative during this period. These patients were transferred to the hospital for a second period of 14 days, in which RT-PCR of the blood, nasopharyngeal swabs, and anal swabs were performed

TABLE 2 | Predictive factors associated with re-positivity for coronavirus nucleic acid in a multivariate analysis.

\begin{tabular}{lccc}
\hline & OR & $\mathbf{9 5 \%} \mathbf{C l}$ & $\boldsymbol{P}$-value \\
\hline Leukopenia & 7.316 & $2.319-23.080$ & 0.001 \\
Hyperpyrexia & 4.643 & $1.426-15.117$ & 0.011 \\
Prealbumin $<15 \mathrm{mg} / \mathrm{dl}$ & 4.199 & $1.461-12.071$ & 0.008 \\
\hline
\end{tabular}

OR, odd ratio; $\mathrm{Cl}$, confidence interval.

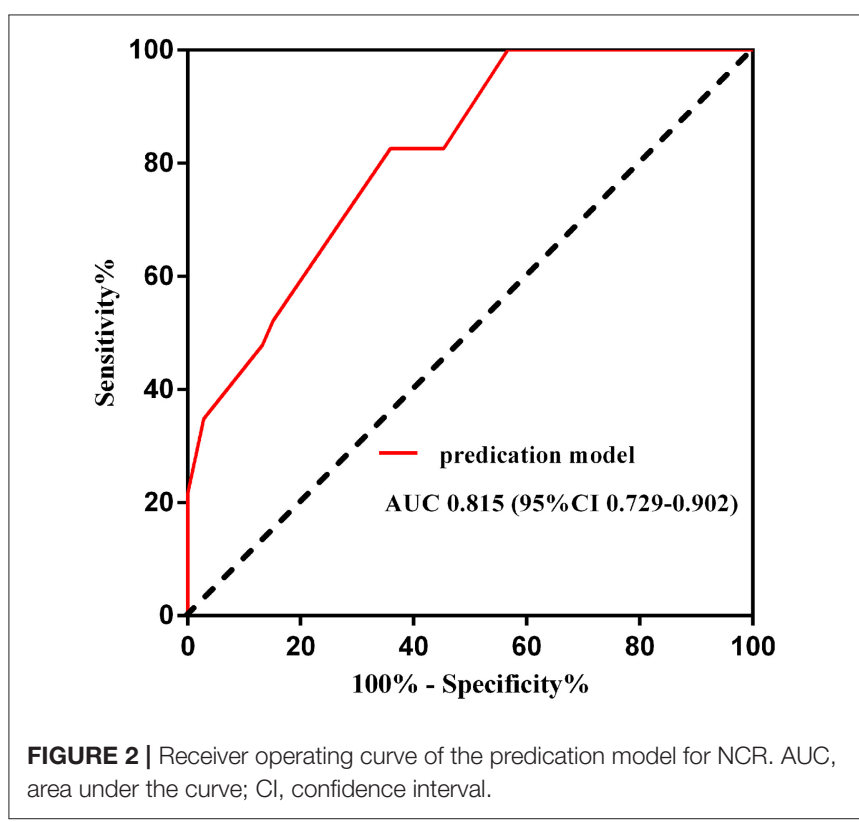


on days $1,4,7$, and 14 . Furthermore, the coronavirus nucleic acid tests of five patients turned negative on day 4,10 patients turned negative on day 7 , and eight patients turned negative on day 14 .

\section{DISCUSSION}

Most COVID-19 patients had a favorable prognosis under "Management of Diagnosis and Treatment of Novel Coronavirus Pneumonia Scheme Trial Version 4." However, the emergence of test re-positivity of SARS-CoV-2 nucleic acid in recovered patients will exacerbate the global situation.

A large study in South Korea showed that 292 (3.3\%) out of 8,922 recovered COVID-19 patients showed re-positivity of virus nucleic acid post-discharge, although there was no detailed description concerning whether all of the recovered patients had been tested or whether only the symptomatic ones had been tested after discharge (11). Yuan et al. (13) reported that the repositivity rate of patients $<18$ years old $(30.8 \%)$ was much higher than that of patients $\geq 18$ years old $(9.5 \%)$. In this study, the repositivity rate was $17.8 \%(23 / 129)$, which was close to the average level reported in previous studies (range: $\sim 14.5-16.7 \%)(9,10$, $12,14)$. To avoid false negatives as much as possible, all patients ( $n=129)$ underwent two consecutive SARS-CoV-2 nucleic acid tests (sampling interval of at least $24 \mathrm{~h}$ ) before discharge.

Hyperexia and low serum prealbumin levels were determined to be independent risk factors associated with test re-positivity of SARS-CoV-2 RNA. Similarly, He et al. (19) reported that the severity of cytokine inflammatory storm was directly related to the severity of disease, as the long-term maintenance of a body temperature above $39^{\circ} \mathrm{C}$ (hyperpyrexia) is a symptom of serious infection. Mahallawi et al. (20) further demonstrated a remarkable pro-inflammatory cytokine response during the acute phase of human MERS-CoV infection. The expression of IFN- $\gamma$, TNF- $\alpha$, IL-15, and IL-17 secreted by pro-inflammatory Th1 and Th17 cells differed significantly between patients with and without this infection. Thus, hyperpyrexia leading to an increased risk of re-positivity of SARS-CoV-2 nucleic acid may be achieved by inducing a cytokine inflammatory storm. Regarding the role of prealbumin in re-positive patients, it was an acute negative time reactive protein similar to albumin, of which the level was significantly lower in COVID-19 patients with a poor prognosis than in those with a good prognosis (21). More time may be required for patients with low serum prealbumin levels to completely eliminate SARS-CoV-2. This may somehow explain why a low serum prealbumin level was associated with test re-positivity for nucleic acid.

Leukopenia was another independent risk factor for repositivity for nucleic acid. Guo et al. (22) indicated that leukocytes, especially lymphocytes (CD3+, CD4+, and CD8+), were significantly reduced in patients who died of viral pneumonia compared with survivors. Changes in the peripheral blood leukocyte count and lymphocyte subsets may play an important role in the pathogenesis of SARS-CoV-2 infection (23). Furthermore, several related studies have also shown that compared with patients with mild COVID-19 infection, the memorability and cytotoxicity of CD8+T cells in severe patients had significantly reduced (1, 20, 24). Lymphocyte apoptosis, immunological injury, and bone marrow suppression may be critical mechanisms leading to lymphopenia observed in severe COVID-19 cases $(25,26)$. However, few studies have demonstrated a relationship between lymphopenia and re-positivity for coronavirus nucleic acid. A comprehensive analysis of the results showed that the clinical manifestations of hyperpyrexia, leukopenia, and low prealbumin levels indicated the tendency for severe SARS-CoV-2 infection. The lymphocyte count in patients with re-positivity was lower than those without re-positivity, although not to a significant degree. This may have been due to the small sample size; hence, large-scale multicenter trials are suggested.

Since all discharged patients followed strict self-isolation protocols, reinfection was relatively unlikely to have led to re-positivity for SARS-CoV2 nucleic acid. All patients with re-positivity were asymptomatic and showed no signs of new pulmonary infiltration on chest CT. Furthermore, none presented with infectivity, and nearly all patients' viral nucleic acid tests turned negative again within a relatively short period (14). The causes of re-positivity of viral nuclear acid might be a false negative nucleic acid test at the time of discharge, or the viral load after treatment is below the lower limit of nucleic acid test. During the isolation period after discharge from the hospital, the viral load increased again, and the nucleic acid re-positivity occurred. Additionally, a recent study (13) suggested that repositivity for SARS-CoV-2 RNA might be considered a process of virus shedding, so the re-positive patients were not infectious. However, different findings were shown in several case reports $(27,28)$, where infectivity was still detected in re-positive patients who had shown multiple negative nasopharyngeal swab tests. Therefore, as there is no clear evidence that re-positive patients cannot transmit the disease, these patients should be followed up scientifically and strictly isolated after discharge to avoid the risk of disease transmission.

However, several limitations must be considered as well. First, this study was retrospective in nature, which is inevitably susceptible for selective bias, observational bias, and confounding bias. Prospective studies concerning COVID-19 are necessary, and propensity score matching (PSM) might be a feasible way to balance out the biases in a retrospective study. Second, this study was conducted at a single center, and the small sample size makes it difficult to generalize the results. Furthermore, we preliminarily demonstrated that leukopenia was an independent risk factor related to re-positivity for SARS-CoV-2 nucleic acid and have yet to subdivide this factor into specific types of white blood cells, such as lymphocytes and neutrophils. To address these shortcomings, further multi-center, large-scale studies are needed.

Immunoglobulin $\mathrm{G}$ (IgG) and immunoglobulin $\mathrm{M}$ (IgM) are two specific serum antibodies for SARS-CoV-2 infection. As the main antibody during the humoral immunity, IgG has a high affinity for the pathogens and is widely distributed in the body, which is also the main force of the body to fight infection. The peak of IgG secretion appears later in infection, but it lasts for a long time. Therefore, IgG is commonly regarded as the main antibody for serological diagnosis and monitoring after 
TABLE 3 | Antibody detection of SARS-CoV-2 viral RNA re-positive patients.

\begin{tabular}{llccc}
\hline & Sex & Age (years) & CoVID-19 & COVID-19 \\
\cline { 4 - 5 } & & & IgG+lgM (S/CO) & IgM (S/CO) \\
\hline Case 1 & Male & 68 & 26.06 & 1.35 \\
Case 2 & Female & 35 & 25.51 & 1.31 \\
Case 3 & Male & 30 & 138.61 & 6.35 \\
Case 4 & Female & 44 & 5.61 & $\mathbf{0 . 4 4}$ \\
Case 5 & Male & 48 & 82.03 & 11.70 \\
Case 6 & Male & 58 & 46.10 & 1.29 \\
Case 7 & Female & 56 & 122.27 & 2.78 \\
Case 8 & Female & 61 & 28.98 & 1.14 \\
Case 9 & Male & 50 & 32.49 & 1.22 \\
Case 10 & Female & 52 & 39.95 & $\mathbf{0 . 2 7}$ \\
Case 11 & Female & 38 & 169.28 & 6.53 \\
Case 12 & Female & 43 & 89.83 & 16.91 \\
Case 13 & Male & 43 & 4.03 & $\mathbf{0 . 2 3}$ \\
Case 14 & Female & 48 & 21.14 & $\mathbf{0 . 6 3}$ \\
Case 15 & Male & 52 & 27.15 & 4.12 \\
\hline
\end{tabular}

IgM, immunoglobulin M; IgG, immunoglobulin G; $S / C O$, sample/cut off; $S / C O<1$ was a negative result; $S / C O \geq 1$ was a positive result for the antibody (Ig G+lgM and IgM). Bold indicates results that are $\mathrm{S} / \mathrm{CO}<1$.

vaccination. However, IgM appears in the early stage of pathogen infection and disappears shortly after acute infection (29). In this study, due to the limited conditions of serum antibody at the early stage of COVID-19 pandemic, viral antibody tests were only performed in a part of re-positive cases $(n=15)$, who were older than other re-positive patients (details in Table 3 ). The serum antibodies (IgG and IgM) of all re-positive patients were significantly increased, especially those of IgG, which indicated that those re-positive patients were in the recovery stage. Moreover, in the follow-up epidemiological investigation, it was found that after all COVID-19 patients $(n=129)$ were cured and discharged from the hospital, no new cases of COVID19 were reported in Zhongxiang City.

In summary, COVID-19 patients with leukopenia, low serum prealbumin levels, and hyperpyrexia are more likely

\section{REFERENCES}

1. Lu R, Zhao X, Li J, Niu P, Yang B, Wu H, et al. Genomic characterisation and epidemiology of 2019 novel coronavirus: implications for virus origins and receptor binding. Lancet. (2020) 395:565-74. doi: 10.1016/S0140-6736(20)30251-8

2. Ren LL, Wang YM, Wu ZQ, Xiang ZC, Guo L, Xu T, et al. Identification of a novel coronavirus causing severe pneumonia in human: a descriptive study. Chin Med J (Engl). (2020) 133:1015-24. doi: 10.1097/CM9.000000000 0000722

3. The species Severe acute respiratory syndrome-related coronavirus: classifying 2019-nCoV and naming it SARS-CoV-2. Nat Microbiol. (2020) 5:536-44. doi: 10.1038/s41564-020-0695-Z

4. Chen N, Zhou M, Dong X, Qu J, Gong F, Han Y, et al. Epidemiological and clinical characteristics of 99 cases of 2019 novel coronavirus pneumonia in Wuhan, China: a descriptive study. Lancet. (2020) 395:507-13. doi: 10.1016/S0140-6736(20)30211-7 to show re-positivity for coronavirus nucleic acid after discharge than others. Although this study was retrospective, single center (Zhongxiang People's Hospital), and had a small sample size $(n=129)$, but with timely and effective treatment, the appropriate extension of hospitalization and the quarantine period may be feasible strategies for managing such patients.

\section{DATA AVAILABILITY STATEMENT}

The raw data supporting the conclusions of this article will be made available by the authors, without undue reservation.

\section{ETHICS STATEMENT}

The studies involving human participants were reviewed and approved by Ethics Committee of Zhongxiang People's Hospital. Written informed consent to participate in this study was provided by the participants' legal guardian/next of kin.

\section{AUTHOR CONTRIBUTIONS}

S-fZ, BS, J-kL, J-cH, J-hL, X-yL, C-wX, HS, and P-lD participated in the clinical treatment. YC, S-wX, X-xW, and S-wL wrote the original draft. P-fL, B-bG, and J-fW undertook validation, writing, review, and editing. All authors contributed to the article and approved the submitted version.

\section{FUNDING}

This work was supported in part by the Program of The National Natural Science Foundation of China (61865014), Natural Science Foundation of Inner Mongolia (NYFY BS2018), Medical Science and Technology Project of Zhejiang Province (2021PY083), Taizhou Municipal Science and Technology bureau (20ywb29, 1701KY22, and 1701KY23), and the Major Research Program of Taizhou Enze Medical Center Grant (19EZZDA2).

5. Clinical findings in a group of patients infected with the 2019 novel coronavirus (SARS-Cov-2) outside of Wuhan, China: retrospective case series. BMJ. (2020) 368:m792. doi: 10.1136/bmj.m792

6. Kobayashi T, Masumoto J, Tada T, Nomiyama T, Hongo K, Nakayama J. Prognostic significance of the immunohistochemical staining of cleaved caspase-3, an activated form of caspase-3, in gliomas. Clin Cancer Res. (2007) 13:3868-74. doi: 10.1158/1078-0432.CCR-06-2730

7. Zhou X, Yang $\mathrm{W}, \mathrm{Li} \mathrm{J}$. Ca2+- and protein kinase C-dependent signaling pathway for nuclear factor- $\mathrm{\kappa} B$ activation, inducible nitricoxide synthase expression, and tumor necrosis factor-alpha production in lipopolysaccharide-stimulated rat peritoneal macrophages. J Biol Chem. (2006) 281:31337-47. doi: 10.1074/jbc.M602739200

8. Lan L, Xu D, Ye G, Xia C, Wang S, Li Y, et al. Positive RT-PCR test results in patients recovered from COVID-19. JAMA. (2020) 323:150203. doi: 10.1001/jama.2020.2783

9. Xing Y, Mo P, Xiao Y, Zhao O, Zhang Y, Wang F. Post-discharge surveillance and positive virus detection in two medical staff recovered from coronavirus 
disease 2019 (COVID-19), China, January to February 2020. Euro Surveill. (2020) 25:2000191. doi: 10.2807/1560-7917.ES.2020.25.10.2000191

10. Qu YM, Kang EM, Cong HY. Positive result of Sars-Cov-2 in sputum from a cured patient with COVID-19. Travel Med Infect Dis. (2020) 34:101619. doi: 10.1016/j.tmaid.2020.101619

11. Kang YJ, South Korea's COVID-19 infection status: from the perspective of re-positive test results after viral clearance evidenced by negative test results. Disaster Med Public Health Prep. (2020) 14:762-3. doi: 10.1017/dmp.2020.168

12. Yuan J, Kou S, Liang Y, Zeng J, Pan Y, Liu L. PCR assays turned positive in 25 discharged COVID-19 patients. Clin Infect Dis. (2020) 714:22302. doi: $10.1093 / \mathrm{cid} / \mathrm{ciaa} 398$

13. Yuan B, Liu HQ, Yang ZR, Chen YX, Liu ZY, Zhang $K$, et al. Recurrence of positive SARS-CoV-2 viral RNA in recovered COVID19 patients during medical isolation observation. Sci Rep. (2020) 10:11887. doi: 10.1038/s41598-020-68782-w

14. Wong J, Koh WC, Momin RN, Alikhan MF, Fadillah N, Naing L. Probable causes and risk factors for positive SARS-CoV-2 test in recovered patients: evidence from Brunei Darussalam. J Med Virol. (2020) 92:284751. doi: $10.1101 / 2020.04 .30 .20086082$

15. Zhu H, Fu L, Jin Y, Shao J, Zhang S, Zheng N, et al. Clinical features of COVID19 convalescent patients with re-positive nucleic acid detection. J Clin Lab Anal. (2020) 34:e23392. doi: 10.1002/jcla.23392

16. Zhang T, Cui X, Zhao X, Wang J, Zheng J, Zheng G, et al. Detectable SARSCoV-2 viral RNA in feces of three children during recovery period of COVID19 pneumonia. J Med Virol. (2020) 92:909-14. doi: 10.1002/jmv.25795

17. Zhu N, Zhang D, Wang W, Li X, Yang B, Song J, et al. A Novel Coronavirus from patients with pneumonia in China, 2019. N Engl J Med. (2020) 382:72733. doi: 10.1056/NEJMoa2001017

18. Wu A, Peng Y, Huang B, Ding X, Wang X, Niu P, et al. Genome composition and divergence of the novel coronavirus (2019-nCoV) originating in China. Cell Host Microbe. (2020) 27:325-8. doi: 10.1016/j.chom.2020.02.001

19. He L, Ding Y, Zhang Q, Che X, He Y, Shen H, et al. Expression of elevated levels of pro-inflammatory cytokines in SARS-CoV-infected ACE2+ cells in SARS patients: relation to the acute lung injury and pathogenesis of SARS. $J$ Pathol. (2006) 210:288-97. doi: 10.1002/path.2067

20. Mahallawi WH, Khabour OF, Zhang Q, Makhdoum HM, Suliman BA. MERS$\mathrm{CoV}$ infection in humans is associated with a pro-inflammatory Th1 and Th17 cytokine profile. Cytokine. (2018) 104:8-13. doi: 10.1016/j.cyto.2018. 01.025

21. Liu W, Tao ZW, Wang L, Yuan ML, Liu K, Zhou L, et al. Analysis of factors associated with disease outcomes in hospitalized patients with
2019 novel coronavirus disease. Chin Med J (Engl). (2020) 133:10328. doi: $10.1097 / \mathrm{CM} 9.0000000000000775$

22. Guo L, Wei D, Zhang X, Wu Y, Li Q, Zhou M, et al. Clinical features predicting mortality risk in patients with viral pneumonia: the MuLBSTA score. Front Microbiol. (2019) 10:2752. doi: 10.3389/fmicb.2019.02752

23. Wang F, Nie J, Wang H, Zhao Q, Xiong Y, Deng L, et al. Characteristics of peripheral lymphocyte subset alteration in COVID-19 pneumonia. $J$ Infect Dis. (2020) 221:1762-9. doi: 10.1093/infdis/jiaa150

24. Xu Z, Shi L, Wang Y, Zhang J, Huang L, Zhang C, et al. Pathological findings of COVID-19 associated with acute respiratory distress syndrome. Lancet Respir Med. (2020) 8:420-2. doi: 10.1016/S2213-2600(20)30076-X

25. Meyre PB, Radosavac M, Baumann L, Piso RJ, Hoffmann M. COVID-19 in a patient with accidental drug-induced neutropenia. Eur J Case Rep Intern Med. (2020) 7:001848. doi: 10.12890/2020_001848

26. Chen RF, Chang JC, Yeh WT, Lee CH, Liu JW, Eng HL, et al. Role of vascular cell adhesion molecules and leukocyte apoptosis in the lymphopenia and thrombocytopenia of patients with severe acute respiratory syndrome (SARS). Microbes Infect. (2006) 8:122-7. doi: 10.1016/j.micinf.2005.06.007

27. Zhang JF, Yan K, Ye HH, Lin J, Zheng JJ, Cai T. SARS-CoV-2 turned positive in a discharged patient with COVID-19 arouses concern regarding the present standards for discharge. Int J Infect Dis. (2020) 97:2124. doi: 10.1016/j.ijid.2020.03.007

28. Bentivegna E, Sentimentale A, Luciani M, Speranza ML, Guerritore L, Martelletti P. New IgM seroconversion and positive RT-PCR test after exposure to the virus in recovered COVID-19 patient. J Med Virol. (2021) 93:97-8. doi: 10.1002/jmv.26160

29. Paterson RW, Brown RL, Benjamin L, Nortley R, Wiethoff S, Bharucha T, et al. The emerging spectrum of COVID-19 neurology: clinical, radiological and laboratory findings. Brain. (2020) 143:3104-20. doi: 10.1093/brain/awaa240

Conflict of Interest: The authors declare that the research was conducted in the absence of any commercial or financial relationships that could be construed as a potential conflict of interest.

Copyright (๔) $2021 \mathrm{Zhu}$, Sun, Li, Cai, Li, Hong, Li, Xu, Li, Xue, Gu, Wu, Zhou, Suo, Duan, $W u$ and Li. This is an open-access article distributed under the terms of the Creative Commons Attribution License (CC BY). The use, distribution or reproduction in other forums is permitted, provided the original author(s) and the copyright owner(s) are credited and that the original publication in this journal is cited, in accordance with accepted academic practice. No use, distribution or reproduction is permitted which does not comply with these terms. 